\title{
Lehren aus der lateinamerikanischen Schuldenkrise
}

\author{
Von Jürgen Westphalen
}

\author{
"Wir können es uns nicht lei- \\ sten, die Fehler der Vergangen- \\ heit zu wiederholen." \\ (US-Finanzminister James A. Baker \\ auf der Jahresversammlung von \\ IWF und Weltbank in Seoul am \\ 8. Oktober 1985)
}

Über Entstehung, Ausmaß und Folgen der Schuldenkrise Lateinamerikas ist in den letzten Jahren so viel geschrieben worden, daß es einer weiteren Darstellung wohl nicht bedarf. Daher ist der Inhalt des folgenden Beitrages darauf begrenzt, zehn besondere Aspekte der Schuldenkrise zu skizzieren und die Folgerungen aufzuzeigen, die sich aus ihnen hinsichtlich der Überwindung der bestehenden bzw. der Vermeidung einer künftigen Krise herleiten lassen.

\section{Krisenbewältigung erfordert langen Atem}

Im August 1982, als das aufstrebende Erdölland Mexiko sich außerstande sah, seinen Schuldendienst auf die im Ausland aufgenommenen Kredite vertragsgemäß zu leisten, ist die lateinamerikanische Schuldenkrise für alle Welt offenkundig geworden. Diesem sogenannten Mexiko-Schock waren für viele lateinamerikanische Länder zwei Jahrzehnte mit beträchtlicher und sich beschleunigender Schuldenaufnahme im Ausland vorausgegangen. ${ }^{1}$ Die öffentlichen Auslandsschulden Lateinamerikas waren in den sechziger Jahren um $155 \%$, in den siebziger Jahren um nicht weniger als $552 \%$ gestiegen.

Es genügt aber nicht, diese Entwicklung bis zur Erdölpreisexplosion Anfang der siebziger Jahre oder darüber hinaus bis in die sechziger Jahre hinein zurückzuverfolgen. Zu einer ernsten Krise ist die Kreditaufnahme Lateinamerikas im Ausland erst dadurch geworden, daß die Grenzen der Schuldendienstkapazität überschritten wurden, die zu einem erheblichen Teil durch die in Jahrzehnten oder sogar Jahrhunderten gewachsenen wirtschaftlichen und sozialen Strukturen der Region bestimmt werden. So manche unbe-

1 Inter-American Development Bank, External Public Debt of the Latin American Countries. Washington 1982. Statistical Abstract. Table No. 1. 
friedigende oder gar falsche Aussage zur Schuldenkrise findet ihre Erklärung darin, daß ihr ein zu enger zeitlicher Horizont zugrunde liegt.

So ist zum Beispiel der häufig zu hörenden Forderung, die Schuldnerländer sollten ihre Kreditwürdigkeit zurückgewinnen und ihren Kapitalbedarf wieder an den internationalen Kapitalmärkten befriedigen, zwar grundsätzlich zuzustimmen; aber es ist - was meistens unterlassen wird - hinzuzufügen, daß dieses Ziel für die Mehrzahl der lateinamerikanischen Länder in sehr weiter Ferne liegt und daß daher auch andere, kürzere Wege gesucht und beschritten werden müssen, die schon bald jedenfalls eine Linderung der Krisenwirkungen erwarten lassen. Ein weiteres Beispiel für eine Fehleinschätzung des zeitlichen Horizonts ist der Ruf nach Schuldenerlaß gewissermaßen als Patentrezept für eine kurzfristige Krisenbewältigung. Schuldenerlaß kann ein durchaus nützliches Instrument sein, aber nur unter der Voraussetzung, daß vorher die Beseitigung der Krisenursachen, insbesondere auch die im allgemeinen nur sehr langfristig realisierbaren strukturellen Anpassungen jedenfalls ernsthaft in Angriff genommen worden sind. Anderenfalls würde mit einer Streichung der Schulden nur eine Atempause gewonnen und die Krise danach wieder in voller Schärfe in Erscheinung treten. ${ }^{2}$ Ein Sonderfall ist der von öffentlichen Kreditgebern gewährte Schuldenerlaß für least developed countries (LDC); dabei handelt es sich indessen in vielen Fällen nur um den Erlaß der Rückzahlung solcher Kredite, die eigentlich von vornherein richtiger als nicht zurückzahlbare Zuschüsse hätten gegeben werden können.

Es ist nicht so sehr die absolute Schuldenhöhe als vielmehr die Besonderheit wichtiger Krisenursachen, die die Bewältigung der Schuldenkrise zu einer äußerst schwierigen Aufgabe und zu einem äußerst langwierigen Prozeß macht. Die Bemühungen um Uberwindung der Krise dürfen sich daher nicht nur auf die Korrektur von Fehlern der letzten zehn oder zwanzig Jahre beschränken, sondern müssen auch die Anpassung von zum Teil in sehr langen Zeiten entstandenen Strukturen an die heutigen wirtschaftlichen und sozialen Gegebenheiten zum Ziel haben.

Die privaten Gläubigerbanken haben diesen Zusammenhang erkannt und sind dementsprechend in ihrer Umschuldungspolitik dazu übergegangen, die Laufzeit für umgeschuldete Kredite erheblich zu verlängern. So betrug zum Beispiel die vereinbarte Laufzeit in der ersten Umschuldungsrunde 1982/1983 für Argentinien 6,8 Jahre, in der vierten Runde 1986/1987 aber bereits 17,6 Jahre; Chile wurde eine Fristverlängerung von 7 auf 15 Jahre, Ecuador von 6,7 auf 18 Jahre und Mexiko von 7,6 auf 19 Jahre zugestanden. ${ }^{3}$

2 Die Problematik des Schuldenerlasses ist dargestellt in: Peter Nunnenkamp, Kapitalabflüsse aus der Dritten Welt und Schuldenerlaß. Zu den Problemen des privaten Kapitaltransfers zwischen Industrie- und Entwicklungsländern. Hrsg. von Institut für Weltwirtschaft, Kiel. Juni 1988.

3 Comisión Económica para América Latina y el Caribe, Balance Preliminar de la Economía Latinoamericana 1987. Santiago de Chile 1987. S. 25. - Comisión Económica para América Latina y el Caribe: Balance Preliminar de la Economía Latinoamericana durante 1985. O.O. 1985. S. 28. 


\section{Prognoseinstrumente und Vorbeugungsmaßnahmen verbessern}

Die Schuldenkrise, die heute die Mehrzahl der lateinamerikanischen Länder belastet, ist nicht aus heiterem Himmel über Schuldner und Gläubiger hereingebrochen. Bereits 1977, fünf Jahre vor dem Mexiko-Schock, war in einer Veröffentlichung der Interamerikanischen Entwicklungsbank ${ }^{4}$ nachzulesen, daß der Schuldendienst auf die öffentlichen Auslandsschulden Lateinamerikas von 1960 bis 1965 um 26,6\%, von 1965 bis 1970 um 41,2 \% und von 1970 bis 1975 um 159,3\% gewachsen war. Diese Zahlen ließen schon damals deutlich erkennen, daß die Region in einen problematischen Engpaß hineinsteuerte. Daraus sind aber weder von Kreditnehmern noch von Kreditgebern Konsequenzen gezogen worden. Im Gegenteil: Im folgenden Jahrfünft von 1975 bis 1980 erhöhte sich die Kreditgewährung aus öffentlichen Quellen im Ausland um $102 \%$ und aus privaten Quellen - vor allem von Geschäftsbanken - um $219 \%$ und infolgedessen stieg der Schuldendienst auf die öffentlichen Auslandsschulden Lateinamerikas um nicht weniger als $302 \% .^{5}$

Der Anstieg von Schulden und Schuldendienst war in den siebziger Jahren, so wird zur Erklärung des damaligen Verhaltens gelegentlich angeführt, begleitet von einer nicht minder kräftigen Steigerung der Exporte, die als die entscheidende Finanzierungsquelle für den Schuldendienst bezeichnet werden. Tatsächlich hat sich in jener Zeit die Schuldendienstquote, also die Relation zwischen Schuldendienstverpflichtung und Exporterlös, für zahlreiche lateinamerikanische Länder nicht verschlechtert. Dabei wird aber übersehen, daß für die Erfüllung der Schuldendienstverpflichtungen nicht der Exporterlös, sondern nur die die Importausgaben übersteigenden Exporteinnahmen zur Verfügung stehen und daß Lateinamerika in den siebziger Jahren erheblich mehr importiert als exportiert hat; die einzigen Jahre mit geringfügigen Exportüberschüssen waren 1973, 1974 und 1979.

Zur Vermeidung künftiger wirtschaftlicher Krisen ähnlichen Ausmaßes ist es heute erforderlich, vorhandene Prognoseinstrumente zu verfeinern und geeignete neue zu entwickeln. Denn wirtschaftliche Entscheidungen bürden im allgemeinen einem Unternehmen oder einer Volkswirtschaft Verpflichtungen in die Zukunft hinein auf. Auch heute noch basieren solche Entscheidungen aber nicht selten auf wirtschaftsstatistischen Daten zum Beispiel vom Internationalen Währungsfonds (IWF), die zwar vertrauenswürdig sein mögen, aber meistens mindestens ein Jahr alt sind und daher nicht das geringste darüber aussagen können, ob die heute eingegangenen Verpflichtungen künftig einmal erfüllbar sein werden oder nicht.

Noch wichtiger ist es aber im Hinblick auf die Bewältigung der gegenwärtigen und auch zur Vorbeugung gegen künftige Krisen, daß schon heute erkennbare Tatsachen zur

4 Banco Interamericano de Desarrollo, El Endeudamiento Externo de América Latina: Situación Actual y Perspectivas. Washington 1977. Cuadro No. 45.

5 Inter-American Development Bank, External Debt of the Latin American Countries. Washington 1986. Table No. 20, 25, 55. 
Kenntnis genommen und auf ihre möglichen künftigen Wirkungen untersucht werden, damit frühzeitig die richtigen Maßnahmen eingeleitet werden können. Solche Fakten und Tendenzen aufzuzeigen, ist Hauptgegenstand dieses Beitrages. Als aktuelle Beispiele für Tatsachen, die zur Kenntnis zu nehmen und aus denen Konsequenzen zu ziehen sind, seien an dieser Stelle angedeutet, daß

- erstens Schuldnerländer wie Bolivien und Chile mit zum Jahresende 1986 ermittelten Auslandsschulden von rd. $70 \%$ ihres Bruttoinlandsprudukts (BIP), Costa Rica mit über $80 \%$ und Nicaragua mit mehr als $200 \%$ bis in das nächste Jahrhundert hinein nicht in der Lage sein werden, ihre Verpflichtungen gegenüber den Gläubigern im Ausland zu erfüllen, ${ }^{6}$ und daß

- zweitens Zinszahlungsverpflichtungen, die sich 1987 für Brasilien auf $35 \%$, für Bolivien auf $40 \%$, für Argentinien auf $56 \%$ und für Nicaragua auf $70 \%$ der Einnahmen aus dem Export von Gütern und Dienstleistungen beliefen, ein auf längere Sicht untragbares Wachstumshemmnis darstellen, zumal von den vier genannten Ländern nur Brasilien einen in Relation zum Zinsendienst angemessenen Exportüberschuß erzielte, während die Handelsbilanzen von Bolivien und Nicaragua mit Passivsalden abschlossen. $^{7}$

Zum Vergleich sei angeführt, daß die von John Maynard Keynes in seiner Schrift "Die wirtschaftlichen Folgen des Friedens" als untragbar bezeichneten "Reparationsleistunge, die Deutschland nach dem Ersten Weltkrieg zu leisten hatte, ... gegen Ende der zwanziger Jahre etwa zwei Prozent des Bruttosozialprodukts, in den schlimmsten Jahren von 1929 bis 1931 ihr Maximum von vielleicht 3,5\% (erreichten). Die Reparationszahlungen machten dabei ungefähr $15 \%$ der Exporterlöse aus. ${ }^{8}$

\section{Verstärkte Anstrengungen unumgänglich}

Eine grundlegende Neuorientierung der Politik zur Uberwindung der Schuldenkrise war von der sogenannten Baker-Initiative erwartet worden, die Inhalt der Rede des amerikanischen Finanzministers James A. Baker auf der Jahresversammlung von Weltbank und IWF am 8. Oktober 1985 in Seoul war. ${ }^{9}$ Das Hauptziel dieser Initiative, die in der Vergangenheit überwiegend restriktiven Anpassungsmaßnahmen wie Importdrosselung, Reduzierung öffentlicher Ausgaben u.ä. weitestmöglich abzulösen durch einen das dauerhafte und möglichst inflationsfreie Wachstum und zugleich auch die außenwirtschaftliche Gesundung fördernde Anpassungspolitik hat allgemeine Zustimmung gefunden.

6 Inter-American Development Bank, Economic and Social Progress in Latin America. 1987 Report. Washington o.J. S. 426, 463.

7 Comisión Económica para América Latina y el Caribe, Balance Preliminar ... 1987. S. $24,21$.

8 André Gunder Frank, Bankrott als Zuflucht. In: Wirtschaftswoche. Nr. 48 (20.11. 1987).

9 Auszugsweise abgedruckt in: Europa-Archiv. Folge 1/1986. S. D 15 ff. 
In den seit der Tagung von Seoul vergangenen Jahren sind allerdings kaum nennenswerte Ergebnisse dieser Initiative zu verzeichnen gewesen:

- Das Wirtschaftswachstum Lateinamerikas, das in den sechziger Jahren im Jahresdurchschnitt 5,4\% und in den siebziger Jahren sogar 6,1\% erreicht hatte, hat sich in den achtziger Jahren merklich verlangsamt, so daß 1987 das Pro-Kopf-Einkommen $5,5 \%$ unter dem Stand von 1980 lag.

- Der Export hat nach einem kräftigen Einbruch 1986 im vergangenen Jahr etwa das Niveau von 1982 wieder erreicht. Der Prozeß der Importdrosselung hat zwar 1984 ein Ende gefunden; aber der bis 1987 erreichte Importwert lag immer noch $33 \%$ unter demjenigen des Jahres 1981, und der Exportüberschuß in der Handelsbilanz betrug im vergangenen Jahr nur $68 \%$ des Ergebnisses von 1985.

- Der gewogene Durchschnitt der Inflationsraten aller lateinamerikanischen Länder ${ }^{10}$ ist von $275 \% 1985$ auf $65 \% 1986$ zurückgegangen, - auf den ersten Blick ein beachtlicher Erfolg. Bei genauerem Hinsehen stellt man aber fest, daß dieses Ergebnis überwiegend mit dem Mittel des Lohn- und Preisstopps erreicht worden ist, das nicht an den Ursachen, sondern an den Symptomen ansetzt. So war es auch nicht überraschend, daß die durchschnittliche Inflationsrate Lateinamerikas schon 1987 wieder auf rd. $220 \%$ emporschnellte.

Die Baker-Initiative zeigt erstrebenswerte Ziele auf, sagt aber zuwenig über die zu deren Erreichung nötigen Maßnahmen und Instrumente. Hinsichtlich der Konkretisierung und Realisierung der Initiative bedarf es in Gegenwart und Zukunft noch erheblich größerer Anstrengungen als bisher. So erscheint es zum Beispiel wenig befriedigend, wenn in der Erklärung der Staats- und Regierungschefs zum Abschluß des Wirtschaftsgipfels in Toronto $^{11}$ die Schuldnerländer mit mittlerem Einkommen, zu denen außer Haiti alle lateinamerikanischen Länder zählen, darauf verwiesen werden, daß "die auf den Einzelfall bezogene marktorientierte Wachstumsstrategie . . nach wie vor der einzige gangbare Weg zur Uberwindung ihrer Schuldenprobleme" ist. Einer Region, deren Pro-Kopf-Einkommen heute noch nicht einmal den Stand von 1980 wieder erreicht hat, müssen auch andere, auf mittlere Sicht aussichtsreiche Wege aus der Krise aufgezeigt werden. Ebensowenig erscheint es ausreichend, "unsere Regierungen", wie es in der zitierten Rede von Minister Baker heißt, "an ihre Verpflichtung . . zu erinnern, protektionistischem Druck zu widerstehen ". Darüber hinaus ist es erforderlich, daß die Wege aufgezeigt und die Schritte getan werden, die zu einem baldigen spürbaren Abbau des für die Schuldnerländer so folgenreichen Importprotektionismus der Industrieländer führen.

10 Comisión Económica para América Latina y el Caribe, Balance Preliminar . . 1987. S. 17.

11 Wirtschaftsgipfel in Toronto. Treffen der Staats- und Regierungschefs vom 19. bis 21. Juni 1988. Politische Erklärung. In: Bulletin Nr. 87 (24. 6. 1988). Hrsg. vom Presse- und Informationsamt der Bundesregierung. 


\section{Importprotektionismus abbauen}

Die Erfüllung der Schuldendienstverpflichtungen setzt eine entsprechende Schuldendienstkapazität voraus, deren Hauptquelle ein aktiver Handelsbilanzsaldo des Schuldnerlandes ist. Die Möglichkeiten vieler Schuldnerländer, ihre Exporteinnahmen zu steigern, werden indessen durch den Importprotektionismus der Industrieländer begrenzt, der mithin die vertragsgemäßen Schuldendienstleistungen unmöglich machen kann. Außerdem bedeuten importprotektionistische Maßnahmen industrialisierter Gläubigerländer auch einen krassen Widerspruch zu deren an die Schulderländer gerichtete Aufforderung, ihre Außenhandelspolitik zu liberalisieren. Auch Finanzminister Baker hat in seiner Rede in Seoul den Schuldnerländern "Maßnahmen zur Marktöffnung mit dem Ziel, ... die Liberalisierung des Handels ... zu fördern", empfohlen.

Diese schlichten Zusammenhänge zwischen Importprotektionismus und Schuldenkrise sowie auch der Widerspruch zwischen Empfehlungen an die Schuldnerländer und eigenem Verhalten sind offenbar von manchen Politikern noch immer nicht klar erkannt worden; anders läßt sich die Kluft zwischen den unzähligen lautstarken Lippenbekenntnissen auf der einen und den wenigen bescheidenen Fortschritten auf der anderen Seite kaum erklären. Zwar haben die Bemühungen des GATT beim Zollabbau zu erf reulichen Fortschritten geführt; stattdessen bedienen sich aber die Industrieländer zunehmend "subtiler und wenig sichtbarer Handelsbarrieren, der nichttarifären Handelshemmnisse" $(\mathrm{NTH})^{12}$, die "besonders die Exportwirtschaft der of t stark außenhandelsabhängigen Entwicklungsländer (treffen), . . . ihren Wirtschaftsaufbau (erschweren) und . . . es den vielfach hochverschuldeten Ländern praktisch unmöglich (machen), die zur Bedienung ihrer Auslandsschulden benötigten Exporterlöse zu erwirtschaften ". ${ }^{13}$

Im Jahre 1981 unterlagen 19\%, 1986 bereits $21 \%$ von der Einfuhr der Industrieländer aus Entwicklungsländern dem sogenannten harten Kern der NTH, zu dem Einfuhrverbote, mengenmäßige Beschränkungen, Export-Selbstbeschränkungsabkommen, die Beschränkungen aufgrund des Welttextilabkommens u.a.m. gehören. ${ }^{14}$

Zutreffend stellen Huss und Nunnenkamp fest, daß nentscheidende Fortschritte (des internationalen Schuldenmanagements) . . . nur zu erzielen (sind), wenn die Industrieländer helfen, die Transferprobleme der Schuldnerländer zu überwinden, indem sie ihre Märkte öffnen" ${ }^{15}$ In der Europäischen Gemeinschaft läßt indessen die für 1992 angestrebte Schaffung eines Wirtschaftsraumes ohne Binnengrenzen eher noch zunehmende Handelsbeschränkungen gegenüber den Drittländern befürchten, zu denen - außer eini-

12 Elisabeth Messner, Freihandel bald nur noch ein Mythos? In: SBG-Wirtschaftsnotizen. Hrsg. von Schweizerische Bankgesellschaft. März 1988.

13 Welthandel durch Protektionismus bedroht. In: Dresdner Bank Außenwirtschaftsnachrichten. 39. Jahrg., Nr. 8. Mitte August 1985.

14 Weltbank, Weltentwicklungsbericht 1987. Washington 1987. S. 159.

15 Hans-Joachim Huss und Peter Nunnenkamp, Finanzinnovationen und Schuldenerlaß - Wege zu einem effizienteren Management der Auslandsschulden von Entwicklungsländern. In: Weltwirtschaft. Halbjahresschrift des Instituts für Weltwirtschaft an der Universität Kiel. 1987, Heft 2. S. 124. 
gen kleinen Staaten in der Karibik - sämtliche lateinamerikanische Länder zählen. Es ist zu hoffen, daß die maßgeblichen Politiker der Gemeinschaft und auch in den EG-Mitgliedsländern der Mahnung von Otto Schlecht, Staatssekretär im Bundesministerium für Wirtschaft, folgen, "die Gemeinschaft (möge) bei der Schaffung des Binnenmarktes der Versuchung... (widerstehen), interne Konflikte durch den scheinbar einfachen Weg der außenwirtschaftlichen Abschottung zu lösen.... Es muß wichtigste Aufgabe der europäischen Handelspolitik bleiben, Hemmnisse für Importe aus Drittländern zu beseitigen, . . . um praktische Entwicklungshilfe zu leisten und Entschuldungsmöglichkeiten für die Dritte Welt zu schaffen". ${ }^{16}$

\section{Umkehr des Ressourcentransfers}

Als Ressourcentransfer wird die Differenz zwischen Nettokapitalzufluß aus dem Ausland und Zahlung von Zinsen und Dividenden an das Ausland bezeichnet. Die Dividenden sind heute - verglichen mit den Zinsen - für die lateinamerikanischen Schuldnerländer von untergeordneter Bedeutung. 1981 ergab sich für Lateinamerika ein Ressourcenzufluß von 10,4 Mrd US-Dollar; für die Zeit von 1982 bis 1987 ist dagegen ein Ressourcenabfluß aus Lateinamerika in Höhe von rd. 146 Mrd US-Dollar bzw. 23\% der gleichzeitigen Exporte von Gütern und Dienstleistungen zu verzeichnen. ${ }^{17}$ Diese Umkehr des internationalen Kapitalstromes beruhte nur "zum geringen Teil auf den gestiegenen Schuldendienstbelastungen; der Kern des Problems lag vielmehr - und liegt noch immer in den drastisch reduzierten Kapitalzuflüssen aus neuen Bankkrediten ". ${ }^{18}$ Allerdings sind in dieser Hinsicht in den lateinamerikanischen Ländern unterschiedliche Entwicklungen zu beobachten: Von dem gesamten Ressourcenabfluß der Region im Jahre 1987 in Höhe von 15,7 Mrd US-Dollar entfielen allein auf Brasilien 48\% und auf Mexiko 28\%, während andererseits acht von neunzehn Ländern einen Ressourcenzufluß hatten.

Im vergangenen Jahr lag der Ressourcenabfluß aus Lateinamerika nicht weniger als $50 \%$ unter demjenigen von 1985, und zwar infolge eines Anstiegs des Nettokapitalzuflusses um 336\% und eines Rückgangs der Zinszahlungen um 13,5\%. Die UN-Wirtschaftskommission für Lateinamerika (CEPAL) ${ }^{19}$ warnt indessen vor voreiligen Folgerungen auf längere Sicht, da die Ursachen der Entwicklung 1986 und 1987 u.a. in der zunehmenden Zahl vorübergehender Moratorien, in der Gewährung neuer Kredite unter dem Druck der Verhältnisse und zur Vermeidung die Gläubiger schädigender Maßnahmen der Schuldnerländer und in der Begrenzung der Zinszahlung auf einen bestimmten An-

16 Otto Schlecht, Der Europäische Binnenmarkt 1992. Herausforderung für Politik und Wirtschaft. Hamburger Beiträge zur Wirtschafts- und Währungspolitik in Europa. Hrsg. von Wilhelm Nölling. Heft 3. Hamburg 1988. S. 25 f.

17 Comisión Económica para América Latina y el Caribe, Balance Preliminar . . 1987. S. 22 f.

18 Peter Nunnenkamp, Kapitalabflüsse ... S. 10.

19 Comisión Económica para América Latina y el Caribe, Balance Preliminar .. 1987. S. 11. 
teil der Exporterlöse gelegen hätten. Maßnahmen dieser Art dienen jedoch, wie die CEPAL feststellt, eher einer Hinausschiebung der Problemlösung als einer dauerhaften Bewältigung der Krise.

Eine Uberwindung der Schuldenkrise auf dem in der Baker-Initiative gewiesenen Weg erfordert eine Umkehr des Ressourcentransfers in einen Ressourcenzufluß aus den Industrieländern in alle lateinamerikanischen Schuldnerländer. Dazu bedarf es einerseits einer weiteren Steigerung des Kapitalzuflusses, die kurzfristig die Schaffung neuer bzw. Verbesserung bestehender Finanzierungsinstrumente und auf lange Sicht die volle Wiederherstellung der Kreditwürdigkeit der Schuldnerländer voraussetzt, und andererseits einer Erleichterung der Zinszahlungsverpflichtung, die vor allem durch eine Veränderung der Quellenstruktur der Auslandsschulden erreicht und überdies durch ergänzende Instrumente wie z.B. einen Zinsausgleichsfonds gefördert werden kann.

\section{Mehr öffentliche Kredite erforderlich}

Die Entstehung der Schuldenkrise ist in den siebziger Jahren nachhaltig durch eine Verschlechterung der Quellenstruktur gefördert worden, d.h. dadurch, daß ein wachsender Anteil der von den Schuldnerländern im Ausland aufgenommenen Kredite aus privaten Quellen, vor allem von Geschäftsbanken, und ein rückläufiger Anteil aus öffentlichen Quellen stammte. 1975 setzten sich die gesamten Auslandsschulden Lateinamerikas, die damals 75,4 Mrd US-Dollar betrugen, folgendermaßen zusammen: 59,2 Mrd US-Dollar bzw. 78,5\% waren Kredite aus privaten Quellen, davon 52,3 Mrd US-Dollar (69,4\% der gesamten Schulden) von Geschäftsbanken, und 16,2 Mrd US-Dollar (21,5\%) stammten aus öffentlichen Quellen. Bis 1981, dem letzteren Jahr vor dem Mexiko-Schock, waren die Gesamtschulden der Region auf 279,7 Mrd US-Dollar gestiegen, die jetzt zu $88,3 \%$ aus privaten Quellen - zu 82,1\% allein von ausländischen Banken - und nur noch zu $11,7 \%$ von öffentlichen Kreditgebern stammten. ${ }^{20}$

Private Kreditgeber müssen sich am Markt refinanzieren und können daher auch nur zu den harten Konditionen des Marktes ausleihen, während aus öffentlichen Quellen, also von multilateralen Instituten wie Weltbank oder Interamerikanischer Entwicklungsbank und von den Regierungen der Industrieländer, auch Kredite zu weichen, d.h. zugunsten des Schuldners von den Marktkonditionen abweichenden Bedingungen gewährt werden können. Die Quellenstruktur der Auslandsschuld eines Landes kann folglich nicht ohne Einfluß auf seine Schuldendienststruktur bleiben: Je höher der Anteil der Schulden aus privaten Quellen an den Gesamtschulden, desto höher auch die Zinszahlungs- im Vergleich zu den Tilgungsverpflichtungen.

Der gesamte Schuldendienst Lateinamerikas gegenüber dem Ausland ist von 12,1 Mrd

20 Inter-American Development Bank, External Debt and Economic Development in Latin America. Background and Prospects. Washington 1984. S. 14, 17. 
US-Dollar 1975 auf 54,0 Mrd US-Dollar 1981 gestiegen. Er setzte sich 1975 zu 49\% aus Zinsen und $51 \%$ aus Tilgungsleistungen zusammen; bis 1981 waren die Zinsen auf $60 \%$ des Schuldendienstes gestiegen und die Tilgungsleistungen auf $40 \%$ zurückgegangen.

Aus dem überdurchschnittlichen Anstieg der Kredite aus privaten Quellen ist der an die Banken gerichtete Vorwurf hergeleitet worden, sie hätten den Kreditrisiken und auch der Kreditverwendung durch die Kreditnehmer nicht genügend Beachtung geschenkt und erheblich mehr Mittel an die Schuldnerländer ausgeliehen, als diese vertragsgemäß zu Marktbedingungen zu bedienen in der Lage wären. In der Tat sind von 1975 bis 1981 die Gesamtschulden Lateinamerikas um 271\% gestiegen, und zwar die Verbindlichkeiten gegenüber öffentlichen Kreditgebern nur um 103\%, gegenüber Banken dagegen um $340 \%$. Besonders zurückhaltend haben sich in jener Zeit die Regierungen der Industrieländer verhalten, deren an lateinamerikanische Länder gegebene Kredite nur um 70\% zunahmen.

Bei einer Beurteilung dieser Kritik an den Banken sollte allerdings nicht übersehen werden, daß damals in den Jahren der Kreditgewährung nur äußerst selten warnende Stimmen zu hören waren und den Banken im Gegenteil immer wieder großes Lob dafür gespendet wurde, daß sie den Prozeß des recycling der Petro-Dollars so routiniert und reibungslos zu bewerkstelligen verstanden. Wie aus heutiger Sicht den Banken ihre zu großzügige Kreditgewährung, so ist den öffentlichen Kreditgebern ihre zu große Zurückhaltung vorzuhalten. Denn es unterliegt keinem Zweifel, daß Quellen- und Schuldendienststruktur der lateinamerikanischen Länder sich günstiger entwickelt hätten, wenn sich auf multilateraler und nationaler Ebene öffentliche Institute in größerem Ausmaß an dem Dollarrecycling beteiligt hätten.

Aus der dargestellten Entwicklung der letzten Jahre zieht die Interamerikanische Entwicklungsbank die sehr weitgehende Folgerung, daß weine weitere Verschuldung aus kommerziellen Quellen zu variablen Zinsen nicht empfohlen werden kann ". ${ }^{21}$ Die angestrebte Umkehr des Ressourcentransfers wird indessen ohne weitere Inanspruchnahme auch der kommerziellen Quellen kaum erreichbar sein. Es muß daher in Zukunft darum gehen, einerseits die aus öffentlichen Quellen zu weichen Konditionen verfügbaren Mittel spürbar zu erhöhen und andererseits die privaten Kreditgeber mittels geeigneter Instrumente in die Lage zu versetzen, ihre Risiken zu mindern und ihre Konditionen zu "entschärfen".

\section{Neue Instrumente entwickeln}

Nach dem Mexiko-Schock vom August 1982 hat die Ergiebigkeit der Finanzierungsquellen Lateinamerikas im Ausland abrupt nachgelassen. 1981 waren die gesamten Auslandsschulden der Region noch um 21,5\% gestiegen, 1983 um 5,4\% und 1985 nur noch

21 Banco Interamericano de Desarrollo, Progreso Económico . . . Informe 1985. S. 109. 
um 2,1\%.22 Entsprechend ist auch der Nettokapitalzufluß nach Lateinamerika, der 1981 mit 37,6 Mrd US-Dollar seinen Höchststand erreicht hatte, 1983 auf nur noch 3 Mrd US-Dollar geschrumpft und nach einer gewissen Erholung im folgenden Jahr 1985 wieder auf 3,3 Mrd US-Dollar zurückgefallen. Auch die Gesamtwirtschaft der Region zeigte nach dem Mexiko-Schock - teils infolge der weltweiten Rezession Anfang der achtziger Jahre, zum Teil aber auch verursacht durch den nachlassenden Kapitalzufluß aus dem Ausland - eine negative Entwicklung: In den Jahren 1982 und 1983 verringerte sich das BIP Lateinamerikas um rd. 3,8\%, das Pro-Kopf-Einkommen um rd. $8 \%$ und der Import um rd. $43 \%$.

Verständlicherweise ist in Lateinamerika die gerade in jener Zeit einer ungünstigen weltwirtschaftlichen Lage besonders nachteilig wirkende Reduzierung der Kapitalversorgung aus dem Ausland beklagt worden. Und den Banken ist vorgehalten worden, dazu mit ihrer gegenüber den siebziger Jahren grundlegend geänderten Politik beigetragen zu haben, die nunmehr zunehmend auf Nichtverlängerung fälliger Kredite und auf beschränkte Neukreditgewährung überwiegend im Rahmen von Umschuldungen ausgerichtet wurde. Dieser Kritik an den Banken ist indessen entgegenzuhalten, daß sich einerseits auch in den Schuldnerländern eine Zurückhaltung gegenüber der Kreditaufnahme zu Marktkonditionen bei privaten ausländischen Instituten breitmachte und daß andererseits die kreditgebenden Banken, nachdem der Mexiko-Schock die Risiken unübersehbar gemacht hatte, auf die veränderte Situation kaum anders als mit äußerst zurückhaltender Neukreditgewährung reagieren konnten.

Die Mobilisierung neuer Kredite aus privaten Quellen wird künftig voraussetzen, daß den Schuldnern tragbare Konditionen, insbesondere feste Zinssätze, eingeräumt und den Kreditgebern zumutbare Risiken geboten werden. Wie belastend variable Zinsen für Kreditnehmer sein können, haben die Schwankungen des LIBOR-Satzes, einer der wichtigsten Orientierungssätze an den internationalen Kapitalmärkten, gezeigt, der sich 1975 im Jahresdurchschnitt auf $8 \%$ belief, 1976 auf 6,3\% sank und $198116,6 \%$ erreichte. ${ }^{23}$ Zur Vermeidung variabler Zinsen in Kreditverträgen mit Entwicklungsländern soll der seit Jahren diskutierte, aber leider noch nicht realisierte Zinsausgleichsfonds ${ }^{24}$ dienen, dessen Mittel von IWF, Weltbank, Regierungen der Industrieländer und privaten Gläubigerbanken aufgebracht werden sollen. Die Vereinbarung von Festzinsen bei der Gewährung neuer Kredite an Entwicklungsländer soll dadurch ermöglicht werden, daß bei einem den Festzinssatz überschreitenden Marktzins die Differenz aus dem Fonds gezahlt wird. Ebenfalls zur Entschärfung der Konditionen, darüber hinaus aber auch zur Risikoeinschränkung zugunsten der privaten Kreditgeber kann die Verfeinerung und der erweiterte Einsatz des schon seit langem von der Weltbank propagierten und auch bereits angewendeten Instruments der Co-Finanzierung durch private und öffentliche Kreditinstitute

22 Inter-American Development Bank, External Debt of the Latin American Countries. Washington 1986. S. 9.

23 Banco Interamericano de Desarrollo, Progreso Económico . . Informe 1985. S. 24.

24 Herrhausen schlägt einen 'Zinsausgleichsfonds` vor. In: Frankfurter Allgemeine Zeitung. Nr. 258/1986. 6. 11. 1986. S. 14. 
beisteuern. ${ }^{25}$ Der Beitrag des öffentlichen Financiers kann teils zugunsten des Schuldners als Zinssubvention und teils zugunsten des privaten Financiers als Garantie gegen Kreditrisiken verwendet werden. Damit können sich unter begrenztem Einsatz öffentlicher Mittel beträchtliche neue Kredite aus privaten Quellen mobilisieren lassen. Zinsausgleichsfonds und Co-Finanzierung sind hier lediglich als Beispiele aus dem weiten Bereich der Verfeinerung bekannter und Entwicklung neuer Instrumente angeführt, in dem künftig noch viel mehr Phantasie und Kreativität als bisher erforderlich sein werden.

\section{Verwendungskontrolle durch öf fentliche Kreditgeber}

Die Geschäftsbanken haben, so ist kritisch bemerkt worden, bei der Vergabe ihrer Kredite an lateinamerikanische Länder in den siebziger Jahren zuwenig auf die Verwendung der Mittel durch die Kreditnehmer gesehen. In der Tat ist in vielen Ländern die Schuldenkrise durch falsche Kreditverwendung erheblich verschärft worden. Die Weltbank ${ }^{26}$ stellt fest, "daß zuviele Investitionen in Projekten vorgenommen wurden, die nicht zu einer deutlichen Steigerung der Produktion führten". In anderen Fällen reagierten Entwicklungsländer auf eine Verschlechterung ihrer Leistungsbilanz damit, daß sie, um eine die Importnachfrage drosselnde Verlangsamung des Wirtschaftswachstums zu vermeiden, ihre Einfuhren durch Schuldenaufnahme im Ausland bezahlten.

Das galt, wie es im Bericht der Weltbank heißt, vor allem für manche Ólimportländer, die ihre nach dem explosionsartigen Ölpreisanstieg 1973 kräftig gewachsenen Leistungsbilanzdefizite mit den damals im Ausland leicht erhältlichen Bankkrediten finanzierten. Nachdem sie diesen bequemen Ausweg einmal mit Erfolg beschritten hatten, versuchten sie es erneut bei dem zweiten Olpreisanstieg Ende der siebziger Jahre. Ein ähnliches Verhalten war aber auch bei Olexportländern zu beobachten, die in den siebziger Jahren infolge hoher Exportpreise beträchtliche Exporteinnahmen erzielten und auch für die achtziger Jahre mit einer weiterhin aufwärts gerichteten Preisentwicklung rechneten. Als sich diese Erwartung nicht erfüllte, setzten sie alles daran, ihre eingeleiteten, zum Teil sehr anspruchsvollen Investitionsprogramme mit im Ausland aufgenommenen Krediten uneingeschränkt fortzuführen. Allgemein bemerkt die Weltbank dazu, daß "viele Regierungen sich verschuldeten, um notwendige Anpassungen im Inland aufzuschieben“. Vielleicht hätten die privaten Banken mehr, als sie es getan haben, auf die Vereinbarung von Verwendungsauflagen drängen und sich um die Uberwachung der Auflagenerfüllung bemühen sollen. Ob aber ein solches Verhalten nennenswerten Erfolg gehabt hätte, ist zu bezweifeln, denn es ist nun einmal ein Wesensmerkmal der Wettbewerbswirtschaft, in die die Banken gestellt sind, daß sich in einer solchen Situation schnell ein Konkurrenzinstitut findet, das das Geschäft für sich zu gewinnen sucht, indem es sich mit weniger

25 Weltbank, Weltenwicklungsbericht 1985. Washington 1985. S. 145.

26 Weltbank, Weltentwicklungsbericht 1985. ...S. 63, 66 f., 76. 
drückenden Konditionen und einer weniger sorgfältigen Uberprüfung der Mittelverwendung zuf riedengibt.

Dagegen haben öffentliche Kreditgeber, insbesondere multilaterale Institute wie Weltbank und IWF, auf diesem Gebiet weitaus größere Wirkungsmöglichkeiten, wie die sogenannte IWF-Konditionalität ungeachtet aller daran geübten Kritik überzeugend gezeigt hat. Für die Zukunft ist daraus ein weiteres Argument für ein wesentlich stärkeres Engagement öffentlicher - nationaler und multilateraler - Institute bei der Deckung des lateinamerikanischen Bedarfs an ausländischen Finanzmitteln zu folgern.

\section{Krisenbewältigung auch bei schwachem Wachstum}

Große Erwartungen im Hinblick auf die Bewältigung der Schuldenkrise werden in die heilsame Wirkung des wirtschaftlichen Wachstums der Industrieländer gesetzt. Im Vorfeld der IWF-Jahrestagung 1984 bezeichnete Bundesfinanzminister Dr. Stoltenberg ${ }^{27}$ "als eine der wichtigsten Voraussetzungen für eine längerfristige Lösung . . . ein dauerhaftes inflationsf reies Wachstum". Nach Stoltenberg ist es entscheidend, "daß die vom IWF für den Zeitraum von 1985 bis 1990 prognostizierten realen Wachstumsraten von $3 \%$ (in den Industrieländern) auch realisiert werden; anderenfalls sähe es 'düster` aus".

Zuversichtlich beurteilte der Bundesverband deutscher Banken ${ }^{28}$ Anfang 1985 die Beziehung zwischen Wirtschaftswachstum der Industrieländer und Schuldenkrise der Entwicklungsländer: "Die Weltwirtschaft befindet sich, von den USA ausgehend, aber nun auch stärker von Europa getragen, in einer Wachstumsphase, die auch 1985 anhalten dürfte. Hiervon werden weiterhin positive Impulse auf Wachstums- und Exportmöglichkeiten der Entwicklungsländer ausstrahlen."

Es besteht kein Zweifel, da ß Wirtschaftswachstum in den Industrieländern auch im Hinblick auf die Lösung der Schuldenkrise wünschenswert ist; es kann allerdings auch die Neigung der Politiker fördern, im Vertrauen auf die Wachstumskräfte die Entwicklung notwendiger neuer Initiativen zu vernachlässigen. Ein Blick auf die tatsächliche Entwicklung zeigt indessen, daß vom Wirtschaftswachstum keine allzu großen Wirkungen zu erwarten sind: In Westeuropa und in den USA war das Wachstum in den letzten Jahren recht gedämpft. In der Europäischen Gemeinschaft schwankte es von 1984 bis 1987 zwischen 2,4\% und 2,6\%, und auch für 1988 ist eine Steigerung der Rate kaum zu erwarten. In den USA ist die 3\%-Marke 1984 deutlich (7,0\%) und 1985 geringfügig $(3,1 \%)$ überschritten worden; in den übrigen Jahren blieb auch die US-Wirtschaft in der "düsteren Sphäre" unterhalb des 3\%-Wachstums, woran sich voraussichtlich auch 1988 nichts ändern wird.

27 Lichtblick in der Verschuldungsproblematik. Bonner Bewertungen im Vorfeld der IMF-Jahrestagung. In: Neue Züricher Zeitung. 31. 8. 1984.

28 Banken zur Internationalen Verschuldung, Ubergang in die zweite Phase im Zeichen der Entspannung. Informationen des Bundesverbandes deutscher Banken. Köln. Januar 1985. 
Bisher haben sich also die Wachstumserwartungen nicht erfüllt, und es sind gegenwärtig keine Anzeichen erkennbar, daß in überschaubarer Zukunft in dieser Hinsicht ein deutlicher und dauerhafter Wandel eintreten könnte. Aber selbst für den Fall, daß künftig in den Industrieländern eine spürbare Wachstumsbeschleunigung erreicht werden sollte, bleiben gewisse Bedenken angebracht:

- Erstens ist zu berücksichtigen, daß die Wirkungen eines solchen Wachstums, die ja hauptsächlich über den Außenhandel auf die Schuldnerländer ausstrahlen, großenteils durch den Importprotektionismus der Industrieländer abgeschwächt, wenn nicht gar zunichte gemacht werden. Die Weltbank ${ }^{29}$ sieht nach wie vor "die große Gefahr, daß die Industrieländer in einer . . . defensiven Weise auf zunehmende Einfuhren von Industrieprodukten reagieren . . . (und damit) die internationale Schuldenproblematik weiter verschärfen".

- Zweitens muß man vor Augen haben, daß wir es bei dem Wachstum der Wirtschaft in den Industrieländern einerseits und der internationalen Schuldenkrise andererseits mit ganz verschiedenen zeitlichen Dimensionen zu tun haben: Mit Sicherheit ist eine so tiefgreifende und weitverbreitete Krise von erheblich längerer Dauer als ein konjunktureller Aufschwung in der industrialisierten Welt.

Daraus ist zu folgern, daß zwar weiterhin alles getan werden sollte, um das Wachstum insbesondere in USA, Westeuropa und Japan zu beschleunigen und die Schuldnerländer daran partizipieren zu lassen; der bei der Beurteilung dieses Zusammenhangs immer wieder zu hörende naive Optimismus sollte indessen einer nüchternen Prüfung der realen Möglichkeiten weichen. Viel intensiver als bisher muß künftig darüber nachgedacht werden, wie sich die Schuldenkrise auch ohne bzw. bei nur schwachem Wachstum bewältigen läßt.

\section{Strukturanpassungen unter Zeitdruck}

Ein u.U. folgenreicher, aber immer wieder anzutreffender Fehler ist es, die Schuldenkrise vorwiegend oder sogar ausschließlich als wirtschaftliches Problem zu betrachten und zu behandeln und ihrem sozialen und politischen Aspekt, von dem schwere Rückwirkungen auf die Wirtschaft ausgehen können, zu wenig Beachtung zu schenken. Die Auslandsverschuldung der lateinamerikanischen Länder ist zwar keinesfalls die alleinige Ursache ihrer sozialen Probleme; ebenso falsch ist es, jeglichen Zusammenhang zwischen Schulden und sozialer Problematik zu ignorieren, denn die Schuldenkrise hat in vielen Ländern erheblich zur Verschärfung dieser Problematik beigetragen. ${ }^{30}$ Dieser Zusammenhang kann hier nur anhand einiger Beispiele angedeutet werden:

29 Weltbank, Weltentwicklungsbericht $1987 . \ldots$. S. 13.

30 Jürgen Westphalen, Schuldenkrise und soziale Probleme in Lateinamerika. In: Europa-Archiv. Folge 23/1986. S. $679 \mathrm{ff}$. 
- Die im Rahmen der Krisenbekämpfung zur Sanierung öffentlicher Haushalte vorgenommene Verringerung öffentlicher Ausgaben für Löhne und Gehälter beeinträchtigt unmittelbar den Lebensstandard vornehmlich der Bezieher niedriger Einkommen.

- Dasselbe gilt für die Aufhebung subventionierter Preise zum Beispiel für Grundnahrungsmittel oder für den Personentransport.

- Eine Inflationsbekämpfung mittels Drosselung der Lohnerhöhungen unter die Rate des Preisanstiegs wirkt nachteilig auf die Realeinkommen der Lohnempfänger.

- Eine so drastische Reduzierung des Imports, wie viele lateinamerikanische Länder sie nach dem Mexiko-Schock erlebt haben, erfaßt großenteils auch die Einfuhr von Produktionsmitteln und dringend benötigten Konsumgütern des täglichen Bedarfs und beeinträchtigt mithin die Beschäftigungsmöglichkeiten und die ausreichende Versorgung vor allem der unteren Einkommensschichten in den betreffenden Schuldnerländern.

- Häufig ist in der Diskussion um die Schuldenkrise die plausibel klingende Feststellung zu hören, die Schuldnerländer hätten in der Vergangenheit über ihre Verhältnisse gelebt und müßten daher heute und in Zukunft den Gürtel enger schnallen. Dabei wird jedoch gewöhnlich übersehen, daß diejenigen, die in der Vergangenheit über ihre Verhältnisse gelebt haben, ganz überwiegend nicht identisch sind mit denjenigen, von denen heute erwartet wird, daß sie ihren Gürtel enger schnallen.

Soziale Unzufriedenheit kann zu politischer Unstabilität führen und den Prozeß der Demokratisierung in Lateinamerika gefährden. Von Mitte der siebziger bis Mitte der achtziger Jahre hat sich die Zahl der diktatorisch regierten lateinamerikanischen Länder von sechzehn auf nur noch drei ${ }^{31}$ verringert. Allerdings kommen Mols und Wolf ${ }^{32}$ in ihrer Untersuchung der Gefährdungselemente, die die Dauerhaftigkeit dieses Prozesses in Frage stellen könnten, zu dem Ergebnis, daß es durchaus noch nicht sicher erscheint, "ob sich der gegenwärtige Redemokratisierungstrend so festigt, daß ein erneuter Umschlag in autoritäre Herrschaftsformen auszuschließen ist".

In Lateinamerika geht es heute darum, ein in Jahrhunderten gewachsenes krasses soziales Gefälle zu glätten, um einerseits die Lage der von den Wirkungen der Schuldenkrise besonders betroffenen armen Bevölkerung zu bessern und damit andererseits die Widerstandsfähigkeit der Demokratien gegen die Erschütterung durch wirtschaftliche und soziale Probleme zu festigen. Dabei können und sollen die Industrieländer helfen, indem sie zum Beispiel dem Bereich der sozialen Anpassung in ihrer Entwicklungshilfe einen besonderen Rang einräumen und überdies, wie der Bundesverband deutscher Banken ${ }^{28}$ zu Recht fordert, bei der Behandlung der Schuldenkrise darauf sehen, daß "die innenund wirtschaftspolitische Belastbarkeit in den jeweiligen Schuldnerländern berücksichtigt" wird.

31 Chile, Kuba, Paraguay.

32 Manfred Mols und Ulrike Wolf, Lateinamerika - Was gefährdet die Demokratie? In: Außenpolitik. Zeitschrift für internationale Fragen. 38. Jahrgang, Nr. 2/1987. S. 208. 
so tıeigreitende und weitverbreitete Krise von erheblich längerer Dauer als ein konjunktureller Aufschwung in der industrialisierten Welt.

Daraus ist zu folgern, daß zwar weiterhin alles getan werden sollte, um das Wachstum insbesondere in USA, Westeuropa und Japan zu beschleunigen und die Schuldnerländer daran partizipieren zu lassen; der bei der Beurteilung dieses Zusammenhangs immer wieder zu hörende naive Optimismus sollte indessen einer nüchternen Prüfung der realen Möglichkeiten weichen. Viel intensiver als bisher muß künftig darüber nachgedacht werden, wie sich die Schuldenkrise auch ohne bzw. bei nur schwachem Wachstum bewältigen läßt.

\section{Strukturanpassungen unter Zeitdruck}

Ein u.U. folgenreicher, aber immer wieder anzutreffender Fehler ist es, die Schuldenkrise vorwiegend oder sogar ausschließlich als wirtschaftliches Problem zu betrachten und zu behandeln und ihrem sozialen und politischen Aspekt, von dem schwere Rückwirkungen auf die Wirtschaft ausgehen können, zu wenig Beachtung zu schenken. Die Auslandsverschuldung der lateinamerikanischen Länder ist zwar keinesfalls die alleinige Ursache ihrer sozialen Probleme; ebenso falsch ist es, jeglichen Zusammenhang zwischen Schulden und sozialer Problematik zu ignorieren, denn die Schuldenkrise hat in vielen Ländern erheblich zur Verschärfung dieser Problematik beigetragen. ${ }^{30}$ Dieser Zusammenhang kann hier nur anhand einiger Beispiele angedeutet werden:

29 Weltbank, Weltentwicklungsbericht 1987 .... S. 13.

30 Jürgen Westphalen, Schuldenkrise und soziale Probleme in Lateinamerika. In: Europa-Archiv. Folge 23/1986. S. 679 ff. 
The article deals with the development, volume, structure and causes of the debt as well as the role of the public and private creditors since the beginning of the crisis. The analysis concludes that the dept burden is a decisive handicap in any effort to restore economic growth and to bring lasting improvements by structural adjustment.

\section{Lessons of the Latin American Debt Crisis}

\section{By Jürgen Westphalen}

The author's reflections begin with the admonition delivered by US Treasury Secretary James A. Baker: "We cannot afford to repeat the mistakes of the past!" Ten aspects of the Latin American debt crisis are used to illustrate misjudgments of specific situations and erroneous reactions of politicians in the early years of the crisis. According to the author, the major problem at present and in the future consists in the fact that on the one hand, the structural adjustment required in order to overcome the debt crisis can only be realized in the long term, while, on the other hand, the avoidance of serious socio-political effects of foreign debt calls for a marked alleviation of the crisis as soon as possible. The question of whether it will be possible for the debt crisis bo be overcome before the region suffers a relapse into dictatorship is regarded as open by the author.

Choice of Law and Forum Clauses in Public External Debt Agreements of Latin American States

\section{By Jürgen Samtleben}

Loan contracts and rescheduling agreements of Latin American states with foreign banks invariably include clauses on jurisdiction and applicable law. Recent events have shown the political and practical importance of such clauses, which usually stipulate the jurisdiction and the law of the bank's home country. The article discusses the validity of such clauses in the light of the Calvo doctrine, of Latin American legislation and of general principles of law. Most of these clauses must be considered unconscionable and therefore invalid. On the other hand, the banks wish to avoid suing the debtor state in its own courts. A solution might be to provide for a forum in a neutral state, or for an arbitral procedure ex aequo et bono regardless of the legislation of any given country, such as stipulated in the loan agreements of the Inter-American Development Bank. 